\title{
Multicritical Behavior in Coupled Directed Percolation Processes
}

\author{
Uwe C. Täuber ${ }^{1, *}$, Martin J. Howard ${ }^{2}$, and Haye Hinrichsen ${ }^{3}$ \\ ${ }^{1}$ Department of Physics - Theoretical Physics, University of Oxford, 1 Keble Road, Oxford OX1 3NP \\ and Linacre College, St. Cross Road, Oxford OX1 3JA, United Kingdom \\ ${ }^{2}$ CATS, The Niels Bohr Institute, Blegdamsvej 17, 2100 Copenhagen Ø, Denmark \\ ${ }^{3}$ Max-Planck-Institut für Physik komplexer Systeme, Nöthnitzer Straße 38, D-01187 Dresden, Germany
}

(July 15, 2018, submitted to Phys. Rev. Lett., OUTP-97-24-S)

\begin{abstract}
We study a hierarchy of directed percolation (DP) processes for particle species $A, B, \ldots$, unidirectionally coupled via the reactions $A \rightarrow B, \ldots$. When the DP critical points at all levels coincide, multicritical behavior emerges, with density exponents $\beta^{(k)}$ which are markedly reduced at each hierarchy level $k \geq 2$. We compute the fluctuation corrections to $\beta^{(2)}$ to $O(\epsilon=4-d)$ using fieldtheoretic renormalization group techniques. Monte Carlo simulations are employed to determine the new exponents in dimensions $d \leq 3$.
\end{abstract}

PACS numbers: 64.60.Ak, 05.40.+j, 82.20.-w.

Nonequilibrium critical phenomena have been the focus of intense theoretical studies in recent years, prominent examples being phase transitions in driven diffusive systems [1], kinetic Ising models [2], nonequilibrium growth models [3], diffusion-limited chemical reactions [4. [. , percolation-like processes [6], and generally in lattice models or cellular automata. The concept of only a few distinct universality classes which determine the critical exponents has been remarkably successful for the description of static and even dynamic critical phenomena near equilibrium phase transitions [7]. However, it is still an unresolved issue whether a similarly small number of universality classes can be identified in nonequilibrium situations, which potentially contain much richer behavior. In addition to the dynamic universality classes listed in Ref. [7], some notable new candidates have emerged in quite different circumstances, for example, the roughening transition in the KPZ equation [8], and the critical point of directed percolation (DP) [9], as described by Reggeon field theory [10]. In fact, the DP universality class appears to cover the majority of phase transitions from non-trivial active into absorbing states where the order parameter noise vanishes. However one striking exception to this rule occurs when the relevant dynamic processes are constrained by an additional local "parity" symmetry, in which case the universality class is that of branching and annihilating random walks with an even number of offspring 11,12.

This work is originally motivated by recent studies of a nonequilibrium growth model for adsorption and desorption of particles which displays a roughening transition in $d=1$ [13]. The key feature is that desorption may take place only at the edge of an existing plateau so that particles cannot be desorbed from a completed layer. Because of this property the dynamic processes at a given height level decouple from all higher levels, i.e. the processes at different heights are unidirectionally coupled. This growth model can in fact be related to a reaction-diffusion problem for a hierarchy of particle species $A, B, \ldots$, which correspond to different height levels, respectively. Each of these species is subject to the prototypical DP processes (see Refs. 10,12])

$$
A \rightarrow A+A, \quad A+A \rightarrow A, \quad A \rightarrow \emptyset,
$$

with reaction rates $\sigma_{A}, \lambda_{A}$, and $\mu_{A}$, respectively (and accordingly for particles $B, \ldots)$. However, there is also a coupling from each previous hierarchy level to the next one via random particle transmutations

$$
A \rightarrow B, \quad B \rightarrow C, \quad \ldots
$$

with rates $\mu_{A B}, \mu_{B C}, \ldots$, but no feedback mechanism from the $B$ to the $A$ species etc. For simplicity, we shall assume that the diffusion constant $D$ for all particle species $A, B, \ldots$ is identical. When $\mu_{A B}=\mu_{B C}=$ $\ldots=0$, the system decouples, and for the $i$-th species there is a critical point separating an active from an absorbing state, where essentially the branching rate $\sigma_{i}$ and decay rate $\mu_{i}$ balance one another. At this point there are three independent critical exponents governing $(i)$ the divergence of the correlation length $\xi \propto\left|r_{i}\right|^{-\nu_{\perp}}$ (where $r_{i} \propto \mu_{i}-\sigma_{i}$ denotes the deviation from the critical point), (ii) the critical slowing down of characteristic time scales $t_{c} \propto \xi^{z} \propto\left|r_{i}\right|^{-\nu_{\|}}$, with $\nu_{\|}=z \nu_{\perp}$, and (iii) the value of the asymptotic particle density in the active phase, $n_{i}(t \rightarrow \infty) \propto\left|r_{i}\right|^{\beta}$. Alternatively, this third independent exponent may be swapped for $\eta_{\perp}$, which characterizes how the equal-time pair correlation function decays at the critical point $r_{i}=0, G(|\mathbf{x}|) \propto 1 /|\mathbf{x}|^{d+z-2+\eta_{\perp}}$ [see Eq. (10) below]. These exponents should be those of the DP universality class, with upper critical (spatial) dimension $d_{c}=4$. Numerical simulations in $d=1$ have confirmed this, and furthermore revealed that $\nu_{\perp} \approx 1.1$ and $z \approx 1.6$ remain unchanged at all hierarchy levels even when the rates $\mu_{A B} \ldots$ are switched on. However, while $\beta_{A}=\beta^{(1)} \approx 0.27$ as in DP for the primary $A$ particles, the particle density exponents for secondary and higher hierarchy levels are considerably reduced to $\beta_{B}=\beta^{(2)} \approx 0.11$, and $\beta^{(3)} \approx 0.04$, provided the critical points for the processes on the different levels coincide [13. 


\begin{tabular}{|l||l|l|l|l|}
\hline \hline & $d=1$ & $d=2$ & $d=3$ & $d=4-\epsilon$ \\
\hline$\beta^{(1)}$ & $0.271(10)$ & $0.57(5)$ & $0.78(7)$ & $1-\epsilon / 6+O\left(\epsilon^{2}\right)$ \\
$\beta^{(2)}$ & $0.108(10)$ & $0.30(4)$ & $0.35(6)$ & $1 / 2-\epsilon / 6+O\left(\epsilon^{2}\right)$ \\
$\beta^{(3)}$ & $0.038(8)$ & $0.13(3)$ & $0.15(4)$ & $1 / 4-\epsilon / 6+O\left(\epsilon^{2}\right)$ \\
\hline \hline
\end{tabular}

TABLE I. Results for the particle density exponents $\beta^{(k)}$ obtained from Monte Carlo simulations and RG calculations.

In the following, we shall mainly study the two-level hierarchy of DP processes (11) for $A$ and $B$ particles coupled by the reaction $A \rightarrow B$. We explain the reduced value of $\beta^{(2)}$ as a consequence of the multicritical behavior that emerges when $r_{A}=r_{B}=r \rightarrow 0$. It appears then rather likely that similar multicritical behavior should emerge in more general contexts than the specific growth model and diffusion-limited chemical reactions (11), (2); namely whenever DP-like processes are coupled unidirectionally without feedback. A qualitatively correct description is obtained by analyzing the corresponding mean-field rate equations, which yield $\beta^{(k)}=1 / 2^{k-1}$ at the multicritical point on the $k$-th hierarchy level. With the aid of the renormalization group (RG), we then identify an additional independent crossover exponent $\phi$ related to the relevant scaling field $\left(\mu_{A B} / D\right)$ of the two-level hierarchy multicritical point. A scaling relation will be derived expressing $\beta^{(2)}$ in terms of $\phi$ and the conventional DP exponent $\beta=\beta^{(1)}$. Using a field-theoretic representation (see Refs. [14,15]) based on the master equation for this stochastic process, we furthermore compute the fluctuation corrections to $\phi$ and $\beta^{(2)}$ to first order in $\epsilon=4-d$ [16]. Finally, these exponents are determined accurately in $d \leq 3$ dimensions by means of Monte Carlo simulations.

We start by writing down the mean-field rate equations for the average local densities $n_{A}(\mathbf{x}, t)$ and $n_{B}(\mathbf{x}, t)$ of the $A$ and $B$ particles, both subject to the reactions (11), and coupled via the random particle transmutation $A \rightarrow B$,

$$
\begin{aligned}
& \partial_{t} n_{A}=D\left(\nabla^{2}-r_{A}\right) n_{A}-\lambda_{A} n_{A}^{2}, \\
& \partial_{t} n_{B}=D\left(\nabla^{2}-r_{B}\right) n_{B}-\lambda_{B} n_{B}^{2}+\mu_{A B} n_{A},
\end{aligned}
$$

where $r_{A}=\left(\mu_{A}+\mu_{A B}-\sigma_{A}\right) / D$ and $r_{B}=\left(\mu_{B}-\sigma_{B}\right) / D$. Considering first the equation for $n_{A}$, it is clear that for $r_{A}>0$ the only stationary state is $n_{A}=0$ (inactive phase). On the other hand, if $r_{A}<0$ the density will asymptotically approach $n_{A}^{\infty}=D\left(-r_{A}\right) / \lambda_{A}>0$ (active state), and hence $\beta^{(1)}=1$ in this approximation. From the diffusive character of Eq. (3), and also from the functional form of its linear term, it follows that $z=2$ and $\nu_{\perp}=1 / 2$ in mean-field. These last two results remain intact for the $B$ particles as well, even in the presence of particle influx via the reaction $A \rightarrow B$. However, the possible stationary states for $n_{B}$ actually depend on the value of $r_{A}$ as well as on $r_{B}$, if $\mu_{A B}>0$. If $r_{A}>0$, and therefore $n_{A}(t \rightarrow \infty)=0$, Eq. (4) reduces to a mean-field DP process with critical point $r_{B}=0$ and $\beta=1$. On the other hand, in the $A$-particle active state, $r_{A}<0$, the stationary solution of (四) becomes $n_{B}^{\infty}=\left[\left(D r_{B} / 2 \lambda_{B}\right)^{2}+\mu_{A B} n_{A}^{\infty} / \lambda_{B}\right]^{1 / 2}-D r_{B} / 2 \lambda_{B}$; i.e., there is merely a crossover from a low-density to a highdensity regime in the vicinity of $r_{B}=0$. An interesting situation arises when $r_{A}=r_{B}=r$, as in this case the second term in the square brackets dominates for $|r| \rightarrow 0$,

$$
n_{B}^{\infty}=\left[D \mu_{A B}(-r) / \lambda_{A} \lambda_{B}\right]^{1 / 2}+O(|r|) .
$$

Hence $\beta^{(2)}=1 / 2$ instead of the mean-field DP value. More generally, this multicritical behavior arises in the regime $\left(D r_{B} / 2 \lambda_{B}\right)^{2} \ll D\left(-r_{A}\right) \mu_{A B} / \lambda_{A} \lambda_{B}$ for $r_{A} \uparrow 0$. On the other hand, for $r_{B}>0$ and $\left(D r_{B} / 2 \lambda_{B}\right)^{2} \gg$ $D\left(-r_{A}\right) \mu_{A B} / \lambda_{A} \lambda_{B}$ one finds $n_{B}^{\infty}=\left(-r_{A}\right) \mu_{A B} / r_{B} \lambda_{A}$, and thus one expects DP transitions for the $B$ species both as $r_{B} \rightarrow 0$ (with $\left.r_{A}>0\right)$ and $r_{A} \rightarrow 0\left(r_{B}>0\right)$ [17.

Notice that there are three critical lines, namely $\left(r_{A}=\right.$ $\left.0, r_{B}>0\right),\left(r_{A}=0, r_{B}<0\right)$, and $\left(r_{A}>0, r_{B}=0\right)$, two of which are critical lines for the $B$ particles, meeting at the point $r_{A}=r_{B}=0$. This special point can therefore be interpreted as a multicritical point, with the meanfield order parameter exponent halved as compared to the ordinary critical point 18$]$. Clearly the generalization of Eqs. (3), (14) to $k$ hierarchy levels leads to $\beta^{(k)}=1 / 2^{k-1}$ at the multicritical point $r_{A}=r_{B}=\ldots=0$.

For $d<d_{c}=4$, one expects that these mean-field values will be strongly modified by fluctuation effects. We have performed Monte Carlo simulations in $d \leq 3$ dimensions in order to determine the survival probability exponent $\beta^{(k)} / \nu_{u}^{(k)}$ up to the third hierarchy level. The DP processes (1) are realized by a cellular automaton which evolves by parallel updates in which a site at time $t+1$ becomes active with probability $p$ provided that the same site, or at least one of its nearest neighbors, was active at time $t$. The coupling (2) between levels is incorporated by the rule that active sites at a given level simultaneously impose active sites on all higher levels. We perform dynamic Monte Carlo simulations [19] where one measures the temporal evolution of the system at criticality starting from a single seed (a single $A$ particle). Assuming that $\nu_{\|}^{(k)}=\nu_{\|}$is identical on all levels $k$, we can use the survival probability to determine the density exponents, giving in $d=1 \beta^{(1)}=0.271(10)$ as in DP [20], $\beta^{(2)}=0.108(10)$, and $\beta^{(3)}=0.038(8)$. These results are consistent with direct estimations of $\beta^{(k)}$ obtained in off-critical simulations [13]. The mean-square spreading exponents yield the exponent $z$, with the results $z^{(1)}=$ $1.57(2)(\mathrm{DP}), z^{(2)}=1.58(2)$ and $z^{(3)}=1.59(2)$, which confirms that these exponents are identical on all levels. Similar results are obtained in $d=2, d=3$ (see Table I).

In order to better understand the very low values found for $\beta^{(k)}$ in the simulations, we clearly have to take fluctuation effects into account. In low dimensions, where reaction noise in the processes (11), (2) is highly important, a straightforward Langevin-type description of the noise can be dangerous, because of the highly non-trivial form of the noise correlations. Instead, it is useful to start 
from the corresponding master equation, and utilize a standard formalism involving a second-quantized bosonic operator representation to derive an effective field theory directly 14, 15]. It is important to note that apart from the continuum limit, this procedure is exact and requires no additional assumptions regarding the precise form of the noise. In the present case, the field theory describing the two-level coupled reactions in $d$ dimensions is defined by the action (we omit terms related to the initial state)

$$
\begin{aligned}
S & =\int d^{d} x \int d t\left\{\bar{a}\left[\partial_{t}+D\left(r_{A}-\nabla^{2}\right)\right] a-\sigma_{A} \bar{a}^{2} a\right. \\
& +\lambda_{A}\left(\bar{a} a^{2}+\bar{a}^{2} a^{2}\right)-\mu_{A B} \bar{b} a \\
& \left.+\bar{b}\left[\partial_{t}+D\left(r_{B}-\nabla^{2}\right)\right] b-\sigma_{B} \bar{b}^{2} b+\lambda_{B}\left(\bar{b} b^{2}+\bar{b}^{2} b^{2}\right)\right\} .
\end{aligned}
$$

We remark that $\langle a\rangle=n_{A}$ and $\langle b\rangle=n_{B}$, whereas the fields $\bar{a}, \bar{b}$ have no direct physical interpretation.

It is now convenient to rescale the fields according to $\bar{a}=\left(\lambda_{A} / \sigma_{A}\right)^{1 / 2} \bar{\psi}_{0}, a=\left(\sigma_{A} / \lambda_{A}\right)^{1 / 2} \psi_{0}, \bar{b}=$ $\left(\lambda_{B} / \sigma_{B}\right)^{1 / 2} \bar{\varphi}_{0}, b=\left(\sigma_{B} / \lambda_{B}\right)^{1 / 2} \varphi_{0}$, and also define new couplings $u_{0}=2\left(\sigma_{A} \lambda_{A}\right)^{1 / 2}, u_{0}^{\prime}=2\left(\sigma_{B} \lambda_{B}\right)^{1 / 2}$, as well as $\mu_{0}=\mu_{A B}\left(\sigma_{A} \lambda_{B} / \sigma_{B} \lambda_{A}\right)^{1 / 2}$ (henceforth, the subscript "0" denotes unrenormalized quantities). If we introduce a length scale $\kappa^{-1}$ and correspondingly measure times in units of $\kappa^{-2}$ (i.e., $\left[D_{0}\right]=\kappa^{0}$ ), we find that the new fields have scaling dimension $\kappa^{d / 2}$, while $\left[r_{A}\right]=\left[r_{B}\right]=$ $\left[\mu_{0}\right]=\kappa^{2}$, which are thus relevant perturbations in the RG sense. On the other hand, $\left[u_{0}\right]=\left[u_{0}^{\prime}\right]=\kappa^{2-d / 2}$, and the corresponding DP nonlinearities become marginal in $d_{c}=4$ dimensions, as expected [10]. It is important to note, however, that $\left[\lambda_{A}\right]=\left[\lambda_{B}\right]=\kappa^{2-d}$, and hence these couplings are irrelevant as compared to $u_{0}$ and $u_{0}^{\prime}$, and may be omitted in the effective action (see Ref. [12]), which consequently reads

$$
\begin{aligned}
S_{\mathrm{eff}} & =\int d^{d} x \int d t\left\{\bar{\psi}_{0}\left[\partial_{t}+D_{0}\left(r_{A}-\nabla^{2}\right)\right] \psi_{0}\right. \\
& -\frac{u_{0}}{2}\left(\bar{\psi}_{0}^{2} \psi_{0}-\bar{\psi}_{0} \psi_{0}^{2}\right)-\mu_{0} \bar{\varphi}_{0} \psi_{0} \\
& \left.+\bar{\varphi}_{0}\left[\partial_{t}+D_{0}\left(r_{B}-\nabla^{2}\right)\right] \varphi_{0}-\frac{u_{0}^{\prime}}{2}\left(\bar{\varphi}_{0}^{2} \varphi_{0}-\bar{\varphi}_{0} \varphi_{0}^{2}\right)\right\} .
\end{aligned}
$$

The saddle-point equations corresponding to this action are precisely the mean-field rate equations (3), (4) but with $\psi_{0}, \varphi_{0}$ instead of $n_{A}, n_{B}$, and with $\mu_{A B}, \lambda_{A}$, and $\lambda_{B}$ replaced by $\mu, u_{0} / 2$, and $u_{0}^{\prime} / 2$. We remark that the harmonic part of this action can be diagonalized only when $r_{A} \neq r_{B}$, leading to new cubic vertices mixing the fields $\psi_{0}$ and $\varphi_{0}$ [compare Eq. (8) below]. However, these additional vertices have no influence on the behavior of the $A$ and $B$ species 21], and the diagonalized theory leads to the anticipated DP critical behavior at both critical lines $\left(r_{A}=0, r_{B} \neq 0\right)$ and $\left(r_{A}>0, r_{B}=0\right)$ [17.

Here we are predominantly interested in the multicritical point $r_{A}=r_{B}=r_{0} \rightarrow 0$, and thus we must work with the non-diagonal action (77). Furthermore, due to the inclusion of the relevant scaling field $\mu_{0}$, a number of "mixed" cubic vertices are additionally generated at the tree level, all of which have scaling dimension $\kappa^{2-d / 2}$. These vertices must be taken into account separately, and hence we need to replace (7) with $S_{\mathrm{mc}}=S_{\mathrm{eff}}+\Delta S$, where

$$
\begin{aligned}
\Delta S=\int d^{d} x \int d t[ & -s_{0} \bar{\varphi}_{0} \bar{\psi}_{0} \psi_{0}-\frac{s_{0}^{\prime}}{2} \bar{\varphi}_{0}^{2} \psi_{0} \\
& \left.+\frac{\tilde{s}_{0}}{2} \bar{\varphi}_{0} \psi_{0}^{2}+\tilde{s}_{0}^{\prime} \bar{\varphi}_{0} \varphi_{0} \psi_{0}\right] .
\end{aligned}
$$

It is now a straightforward task to compute the renormalized couplings and the RG fixed points to one-loop order. The DP nonlinearities in (7) remain unaffected by the "mixed" vertices (8), and the stable nontrivial fixed points for the associated dimensionless renormalized couplings $u=u_{0} A_{d}^{1 / 2} \kappa^{(d-4) / 2}$ and $u^{\prime}=u_{0}^{\prime} A_{d}^{1 / 2} \kappa^{(d-4) / 2}$, where $A_{d}=\Gamma(3-d / 2) / 2^{d-1} \pi^{d / 2}$, are

$$
\left[(u / D)^{*}\right]^{2}=\left[\left(u^{\prime} / D\right)^{*}\right]^{2}=4 \epsilon / 3+O\left(\epsilon^{2}\right) .
$$

Hence the critical exponents $\eta_{\perp}=-\epsilon / 12+O\left(\epsilon^{2}\right), \nu_{\perp}^{-1}=$ $2-\epsilon / 4+O\left(\epsilon^{2}\right)$, and $z=2-\epsilon / 12+O\left(\epsilon^{2}\right)$ remain those of the DP universality class at all hierarchy levels, whereas the exponent $\beta^{(k)}$ is unchanged only at the first level

$$
\beta^{(1)}=\frac{\nu_{\perp}}{2}\left(d+z-2+\eta_{\perp}\right)=1-\epsilon / 6+O\left(\epsilon^{2}\right) .
$$

For the similarly defined renormalized "mixed" cubic vertices, one finds two RG fixed lines. The first is given by

$$
\begin{gathered}
(s / D)^{*}=-\left(\tilde{s}^{\prime} / D\right)^{*},(\tilde{s} / D)^{*}-\left(s^{\prime} / D\right)^{*}=2(s / D)^{*} \\
{\left[(s / D)^{*}\right]^{2}=2(\epsilon / 3)^{1 / 2}\left[(s / D)^{*}+\left(s^{\prime} / D\right)^{*}\right]}
\end{gathered}
$$

with stability matrix eigenvalues $0,0,-\epsilon / 3,-\epsilon / 3$, which imply that this fixed line (including the Gaussian fixed point for $\Delta S$ ) is unstable for $d<4$. The second fixed line,

$$
\left(s^{\prime} / D\right)^{*}=(\tilde{s} / D)^{*},(s / D)^{*}+\left(\tilde{s}^{\prime} / D\right)^{*}=2(\epsilon / 3)^{1 / 2},
$$

with again Eq. (12), turns out to be stable as its stability matrix has eigenvalues $0, \epsilon / 3, \epsilon / 3,4 \epsilon / 3$ [17]. We remark that both fixed lines (11) and (13) with (12) satisfy the condition $(\epsilon / 3)^{1 / 2}\left[\left(s^{\prime} / D\right)^{*}+(\tilde{s} / D)^{*}\right]=-(s / D)^{*}\left(\tilde{s}^{\prime} / D\right)^{*}$, which ensures the cancellation of strongly singular (UVdivergent in $d=2$ ) diagrams for the renormalization of $\mu_{0}$. The RG eigenvalue of $\mu$ at these fixed lines then becomes $y_{\mu}=2+\epsilon / 6$, and $y_{\mu}=2-\epsilon / 6$, respectively. In principle a product of quartic vertices and $\mu_{0}$ might enter the renormalizations of the three-point functions as well. However, we have checked that these additional couplings all have negative RG eigenvalues and are therefore irrelevant [17].

We next define the crossover exponent $\phi$ related to the new scaling field $\mu / D$, as it appears in the general scaling form for the $B$-species "density" field,

$$
\varphi(|r|, \mu / D)=|r|^{\beta^{(1)}} \hat{\varphi}\left(|r|^{-\phi} \mu / D\right)
$$


and identify

$$
\phi=\nu_{\perp}\left(y_{\mu}+z-2\right)=1+O\left(\epsilon^{2}\right)
$$

at the stable fixed line.

Finally, we relate the above exponent $\phi$ to the density exponent $\beta^{(2)}$ on the second hierarchy level. We first use the RG flow equations for the scale-dependent renormalized parameters, and then match to our earlier mean-field result (5) for the density in the active phase in the vicinity of the multicritical point. In terms of the rescaled fields, we found $\varphi_{0}^{\infty}=\left(2 \mu_{0} \psi_{0}^{\infty} / u_{0}^{\prime}\right)^{1 / 2}$ for $r_{0} \rightarrow 0$. Essentially, we now have to replace the bare parameters here by their flowing renormalized counterparts, when inverse length scales are rescaled according to $\kappa \rightarrow \kappa \ell$. To that end, we first note that the fields $\psi$ and $\varphi$ scale as $\ell^{\left(d+z-2+\eta_{\perp}\right) / 2}$ [which, with the matching condition $\ell=(-r)^{\nu_{\perp}}$, actually implies the scaling relation cited in Eq. (10)]. Furthermore, the $O(\epsilon)$ fixed points (9) require $u^{\left({ }^{\prime}\right)}(\ell) / D(\ell) \rightarrow$ const., and according to our definitions, we have $r(\ell) \rightarrow r \ell^{-1 / \nu_{\perp}}$ and $\mu(\ell) / D(\ell) \propto \ell^{-\phi / \nu_{\perp}}$. Upon identifying $\ell=(-r)^{\nu_{\perp}}$, we finally arrive at

$$
\beta^{(2)}=\beta^{(1)}-\phi / 2=1 / 2-\epsilon / 6+O\left(\epsilon^{2}\right),
$$

using our above results [16]. Equivalently, this follows by demanding that the scaling function in Eq. (14) behave as $\hat{\varphi}(x) \rightarrow x^{1 / 2}$ for $x \rightarrow \infty$, as prescribed by mean-field theory (5). The $O(\epsilon)$ result (16) constitutes a sizable downward renormalization through fluctuation effects, as is also evident from the simulation data in Table I. These can be explained with the values $\phi \approx 0.33,0.54$, and 0.86 in $d=1,2$, and 3 , respectively.

In principle, these considerations are readily generalized to further hierarchy levels [17]. For example, although a combination of processes $A \rightarrow B$ and $B \rightarrow C$ will generate the reaction $A \rightarrow C$ as well, one finds that the additional relevant scaling field $\mu_{A C}$ does not enter the leading contribution to the $C$ density near the multicritical point $r_{A}=r_{B}=r_{C}=r \rightarrow 0$. Hence $n_{C} \approx\left[D \mu_{A B} \mu_{B C}^{2}(-r) / \lambda_{A} \lambda_{B} \lambda_{C}^{2}\right]^{1 / 4}$, implying that no additional independent exponents need to be introduced. We then find $\beta^{(3)}=\beta^{(1)}-3 \phi / 4=1 / 4-\epsilon / 6+O\left(\epsilon^{2}\right)$.

In summary, we have studied a hierarchy of unidirectionally coupled DP processes (no feedback), as defined by the diffusion-limited reactions (1), (2). Already within mean-field theory, we have identified a purely dynamic multicritical point, which occurs when the DP critical points at each level coincide. Using a field-theoretic representation, we have evaluated to $O(\epsilon)$ the crossover exponent $\phi$ related to the relevant scaling field $\mu_{A B} / D$. We then derived a scaling relation which allowed us to determine $\beta^{(2)}$. In accord with our Monte Carlo simulation results, fluctuation effects lead to a strong downward renormalization of the density exponents as compared with the mean-field values $\beta^{(k)}=1 / 2^{k-1}$. We emphasize that the existence of such a multicritical regime is to be expected generically whenever processes of the ubiquitous DP universality class are coupled unidirectionally.
We benefited from discussions with J.L. Cardy, M.R. Evans, Y.Y. Goldschmidt, and D. Mukamel. U.C.T. acknowledges support from the European Commission, contract No. ERB FMBI-CT96-1189, and from the Deutsche Forschungsgemeinschaft, Gz. Ta 177 / 2.

* Present address: Institut für Theoretische Physik, Physik-Department der TU München, James-FranckStraße, D-85747 Garching, Germany.

[1] B. Schmittmann and R.K.P. Zia, in Phase Transitions and Critical Phenomena, eds. C. Domb and J.L. Lebowitz (Academic Press, New York, 1996).

[2] M. Droz, Z. Rácz, and J. Schmidt, Phys. Rev. A 39, 2141 (1989).

[3] A.L. Barabási and H.E. Stanley, Fractal Concepts in Surface Growth (Cambridge University Press, Cambridge, 1995); J. Krug, Adv. Phys. 46, 139 (1997).

[4] V. Kuzovkov and E. Kotomin, Rep. Prog. Phys. 51, 1479 (1988).

[5] J.L. Cardy, in Proceedings of mathematical beauty of physics, ed. J.-B. Zuber, Adv. Ser. in Math. Phys., vol. 24, to appear (report cond-mat/9607163).

[6] Percolation Structures and Processes, ed. G. Deutscher, R. Zallen, and J. Adler, Ann. Isr. Phys. Soc. 5 (Adam Hilger, Bristol, 1983).

[7] P.C. Hohenberg and B.I. Halperin, Rev. Mod. Phys. 49, 435 (1977).

[8] M. Kardar, G. Parisi, and Y.-C. Zhang, Phys. Rev. Lett. 56, 889 (1986); for reviews, see Ref. [3] and T. HalpinHealy and Y.-C. Zhang, Phys. Rep. 254, 215 (1995).

[9] For a review, see W. Kinzel, in Ref. [6], p. 425.

[10] P. Grassberger and K. Sundermeyer, Phys. Lett. 77 B, 220 (1978). J.L. Cardy and R.L. Sugar, J. Phys. A 13, L423 (1980); H.K. Janssen, Z. Phys. B 42, 151 (1981).

[11] P. Grassberger, F. Krause, and T. van der Twer, J. Phys. A 17 L105 (1984); H. Takayasu and A.Yu. Tretyakov, Phys. Rev. Lett. 68, 3060 (1992); N. Menyhárd and G. Ódor, J. Phys. A 28, 4505 (1995); K.E. Bassler and D.A. Browne, Phys. Rev. Lett. 77, 4094 (1996); H. Hinrichsen, Phys. Rev. E 55, 219 (1997).

[12] J.L. Cardy and U.C. Täuber, Phys. Rev. Lett. 77, 4780 (1996); preprint, to appear in J. Stat. Phys. 90 (1998).

[13] U. Alon, M.R. Evans, H. Hinrichsen, and D. Mukamel, Phys. Rev. Lett. 76, 2746 (1996); report condmat/9710142.

[14] M. Doi, J. Phys. A 9, 1479 (1976); P. Grassberger and P. Scheunert, Fortschr. Phys. 28, 547 (1980); L. Peliti, J. Phys. (Paris) 46, 1469 (1984); B.P. Lee, J. Phys. A 27, 2633 (1994).

[15] B.P. Lee and J.L. Cardy, J. Stat. Phys. 80, 971 (1995); M.J. Howard and J.L. Cardy, J. Phys. A 28, 3599 (1995); P.-A. Rey and M. Droz, J. Phys. A 30, 1101 (1997); M.J. Howard and U.C. Täuber, J. Phys. A 30, 7721 (1997).

[16] The one-loop results were also independently derived by Y.Y. Goldschmidt (unpublished, private communication). 
[17] Further details, as well as some subtleties of the theory in the active phase (Y.Y. Goldschmidt, private communication) will be presented elsewhere: U.C. Täuber, M.J. Howard, H. Hinrichsen, and Y.Y. Goldschmidt (unpublished).

[18] This is remarkably similar to the equilibrium tricritical behavior in the $\left(r \Phi^{2}+u \Phi^{4}+v \Phi^{6}\right)$-model, where $\beta=1 / 2$ for $u>0$ and $\beta=1 / 4$ for $u=0$ in the Landau approximation. Notice, however, that the mechanism leading to the reduction of the exponent $\beta$ is quite different here.

[19] P. Grassberger and A. de la Torre, Ann. Phys. (N.Y.) 122, 373 (1979).

[20] I. Jensen and A.J. Guttmann, J. Phys. A 28, 4813 (1995); I. Jensen, Phys. Rev. Lett. 77, 4988 (1996).

[21] H.K. Janssen, Phys. Rev. Lett. 78, 2890 (1997). 